\title{
Android Phone Speech Recognition Sensed Voice Operated Notice Board Display
}

\author{
Rajat Agarwal $^{1}$, Sanjeev Singh ${ }^{2}$, Sharad Yadav ${ }^{3}$, Shubham Bansal ${ }^{4}$ \\ U.G. Students, Dept of E\&C Engg, MIT Moradabad Ram Ganga Vihar, Phase II, Moradabad, India 1,2,3,4
}

\begin{abstract}
The notice boards are important thing to publish the notices in institutes/organization or public places like hospitals, railway stations, bus stands. The Notice boards are used to display the information in an effective way to the people. Now a day Mostly notice board are of paper sticking type but to use the paper sticking type notice board is wastage lot of time, paper and labour and using the paper notices, it is not easy to update the messages instantly on the notice board. It is tried to overcome these drawbacks in this project. This project deals about an advanced Hi-Tech wireless Notice Board. This system is enhanced to display the latest information through an Android application of smart phones or tablet.
\end{abstract}

Keywords: Speech Recognition, Sensed Voice, Android Application, Smart Phones, Hi-Tech wireless Notice Board.

\section{INTRODUCTION}

The main objective of the project is to develop a wireless notice board that displays notices when a voice message is sent from the users mobile using an android app. While the user sends the message from the mobile, the remote operation is achieved by any smart-phone/Tablet etc., with Android OS, upon a GUI (Graphical User Interface) based voice operation. Bluetooth wireless technology is a popular technique in the communication arena, and it is one of the fastest growing fields in the wireless technologies. Bluetooth technology handles the wireless part of the communication channel; it is used in this project to transmit and receive data wirelessly between devices. While a phone is simply more than a phone these days, it is a smartphone the number of applications being built on a wide range of platforms for smart phone is astounding.

Transmitting end uses an Android application device remote through which commands are transmitted. At the receiver end, these commands are converted to texts used which are displayed on a LED Display interfaced to the Arduino. Serial communication data sent from the Android application is received by a Bluetooth receiver interfaced to the Arduino.

\section{LITERATURE SURVEY}

\section{[1.]Abhishek Gupta[GSM BASED WIRELESS NOTICE BOARD]}

In this project, mainly focused on the GSM technology, Raspberry pi technology and LCD monitor. In this world everyone needs a comfortable living life. Man has researched different technology for his sake of life. In today's world of connected ness, people are becoming accustomed to easy access to information. Whether it's through the internet or television, people want to be informed and up-to-date with the latest events happening around the world. Wired network connection such as Ethernet has many limitations depending on the need and type of connection. Now a day's people prefer wireless connection because they can interact with people easily and it require less time.

\section{[2.]Neenu Ann George [Raspberry Pi Based Speech Recognition Sensed Smart Notice Board Display]:}

This project introduces an innovative android based notice board display system that allows the user to display notices without typing them manually. Here the announcer may speak out the message through an android phone which uses a developed speech to text mobile application. The text message is then transferred via E-mail using Wi-Fi Dongle or Ethernet to Raspberry pi and is then displayed on the HD Display screen. To demonstrate this concept, any display that can be connected to HDMI port of Raspberry pi can be used to display messages. This innovative system can be used in a variety of places including railway stations, schools, colleges, offices for displaying emergency announcements on the screen instantly by just speaking out the message instead of typing it in each time.

\section{[3.]Ramchandra K. Gurav[Wireless Digital Notice Board Using GSM Technology]:}

In this system the message send from authorized user to GSM module which is located on the notice board. So this GSM module receives the message and displayed on notice board, at same time this message will be send different mobile 
number store in memory of microcontroller. When new message is arrived at notice board than the buzzer will beep. Max232 shift the level of signal which converts the signal between the microcontroller and GSM module. After the conversion of signal this message will be displayed on notice board.

\section{[4.]Foram Kamdar[Display Message on Notice Board using GSM]:}

This project deals with the GSM module.It presents an SMS based notice board incorporating the widely used GSM to facilitate the communication of displaying message on notice board via user's mobile phone. Its operation is based on microcontroller ATMEGA32 programmed in assembly language. A SIM300 GSM modem with a SIM card is interfaced to the ports of the microcontroller with the help of AT commands. When the user sends a SMS via a registered number from his mobile phone, it is received by SIM300 GSM modem at the receiver's end. SIM300 is duly interfaced through a level shifter IC MAX32 to the microcontroller. The messaged is thus fetched into the microcontroller. It is further displayed on an electronic notice board which equipped with LCD display interfaced to microprocessor powered by a regulated power supply from mains supply of 230 volts ac.

\section{CURRENT MODELS OF NOTICE BOARDS}

Currently we rely on putting up notices on the notice boards using papers. This is time consuming and also there is wastage of paper. If we need to renew the notice then we have to take a new hard copy. A separate person is required to take care of this notice display. Now a days GSM modem based notice boards are also in use but they require router in which cable connections are done which make it complex.

\section{PROPOSED WORK}

In view of the current models of notice board it will be apparent that, there exists a need of electronic notice board that enables efficient way to the user for displaying notice. This project is an implementation of the idea of wireless communication between a mobile phone and an Arduino. The display unit consists of LED display that is interfaced with arduino. Bluetooth is an open wireless protocol for exchanging data over short distances from fixed and mobile devices, creating Personal Area Networks (PANs). It was originally conceived as a wireless alternative to RS232 data cables. It can connect several devices, overcoming problems of synchronization.

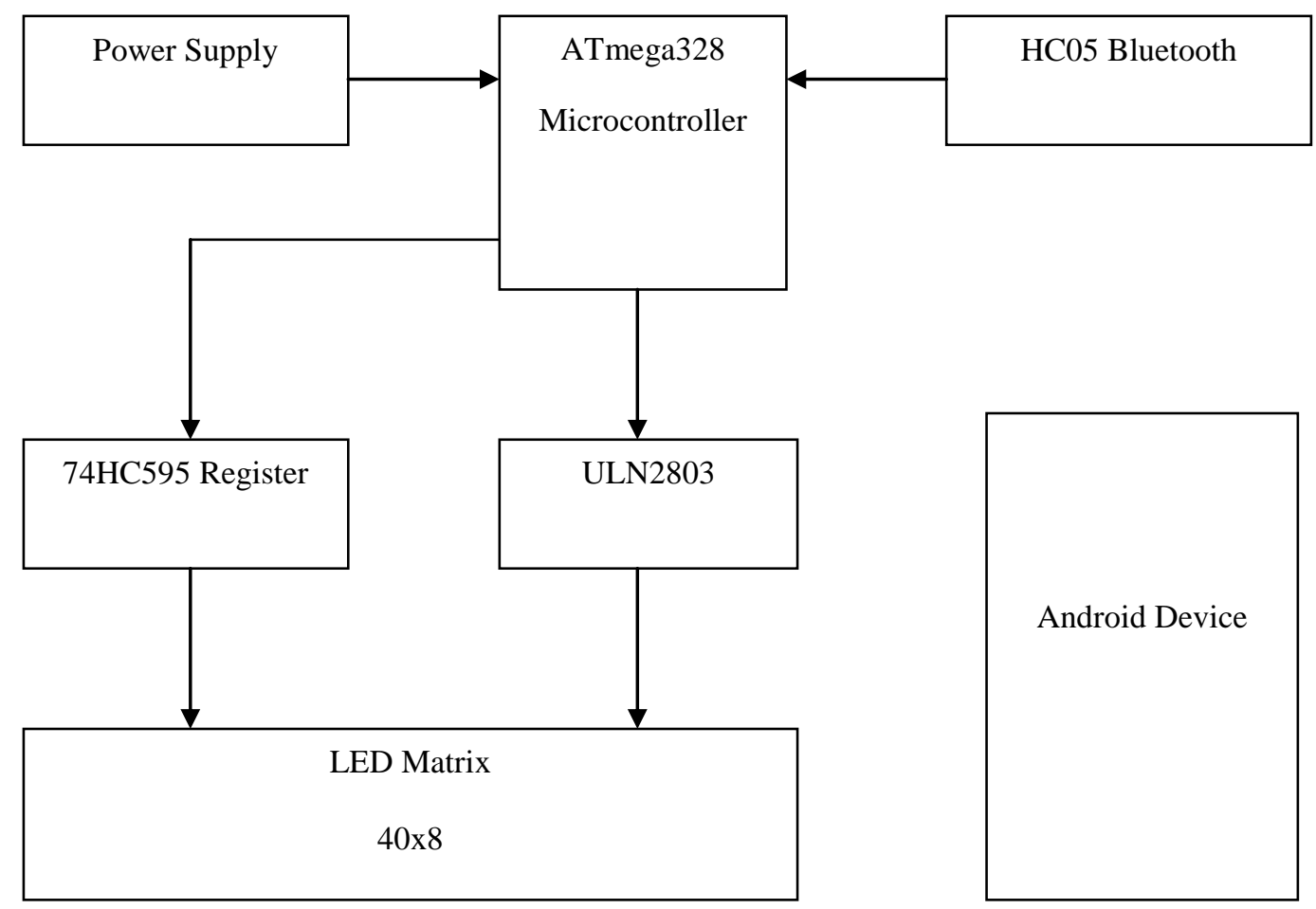


Bluetooth will receive the signal sent by the Android application device (mobile phone), and then send this signal to the Arduino. In order to implement this project, we need to create an Android application that is capable of performing the following

Functions:

- Convert voice data to text

- Send this text over to arduino via

- Bluetooth for displaying on notice board

\section{ATmega328p:}

As of 2013 the ATmega328 is commonly used in many projects and autonomous systems where a simple, low-powered, low-cost micro-controller is needed. Perhaps the most common implementation of this chip is on the popular Arduino development platform, namely the Arduino UNO and Arduino Nano models.Reliability qualification shows that the projected data retention failure rate is much less than $1 \mathrm{PPM}$ over 20 years at $85^{\circ} \mathrm{C}$ or 100 years at $25^{\circ} \mathrm{C}$.

The Atmel 8-bit AVR RISC-based micro controller combines $32 \mathrm{~KB}$ ISP flash memory with read- while-write capabilities,1 KB EEPROM, 2 KB SRAM, 23 general purpose I/O lines, 32 general purpose working registers, three flexible timer/counters with compare modes, internal and external interrupts, serial programmable USART, a byte-oriented 2-wire serial interface, SPI serial port, 6-channel 10-bit A/D converter (8-channels in TQFP and QFN/MLF packages), programmable watchdog timer with internal oscillator, and five software selectable power saving modes. The device operates between 1.8-5.5 volts. The device achieves throughput approaching 1 MIPS per MHz.

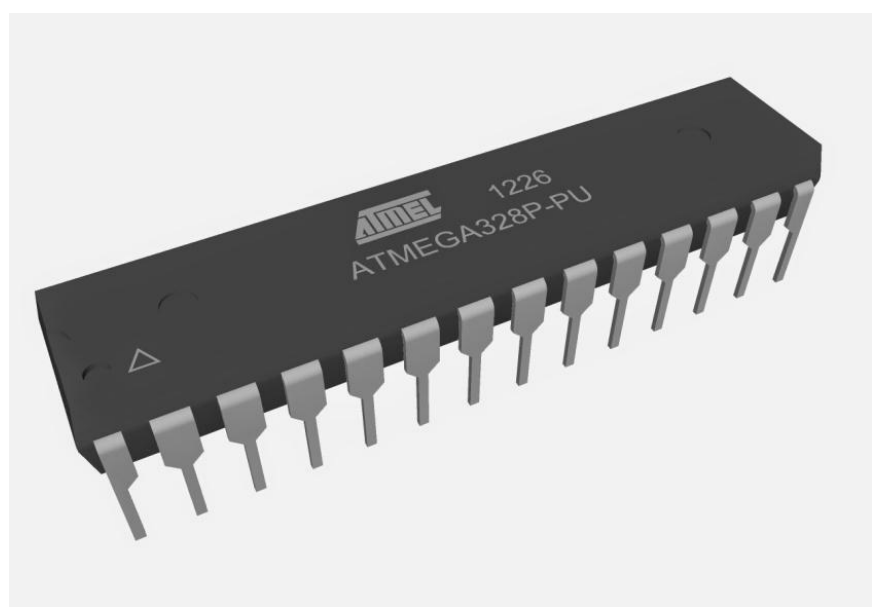

\section{BLUETOOTH MODULE:}

A BlueTooth module is usually a hardware component that provides. a wireless product to work with the computer; or in some cases, the. bluetooth may be an accessory or peripheral, or a wireless headphone. or other product (such as cellphones can use.) If the computer (is this computer related?) has hardware support to.

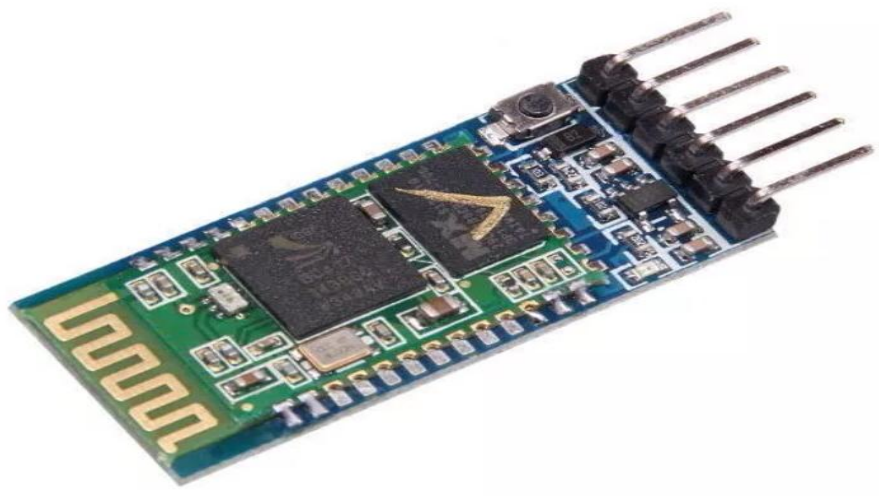


HC-05 module is an easy to use Bluetooth SPP (Serial Port Protocol) module, designed for transparent wireless serial connection setup. Serial port Bluetooth module is fully qualified Bluetooth V2.0+EDR (Enhanced Data Rate) 3Mbps Modulation with complete $2.4 \mathrm{GHz}$ radio transceiver and baseband. It uses CSR Bluecore 04- External single chip Bluetooth system with CMOS technology and with AFH(Adaptive Frequency Hopping Feature). It has the footprint as small as $12.7 \mathrm{~mm} \times 27 \mathrm{~mm}$.

\section{THE 74HC595 SHIFT REGISTER:}

The shift register holds what can be thought of as eight memory locations, each of which can be a 1 or a 0 . To set each of these values on or off, we feed in the data using the 'Data' and 'Clock' pins of the chip. The clock pin needs to receive eight pulses, at the time of each pulse, if the data pin is high, then a 1 gets pushed into the shift register, otherwise a 0 . When all eight pulses have been received, then enabling the 'Latch' pin copies those eight values to the latch register. This is necessary, otherwise the wrong LEDs would flicker as the data was being loaded into the shift register. The chip also has an $\mathrm{OE}$ (output enable) pin, this is used to enable or disable the outputs all at once.

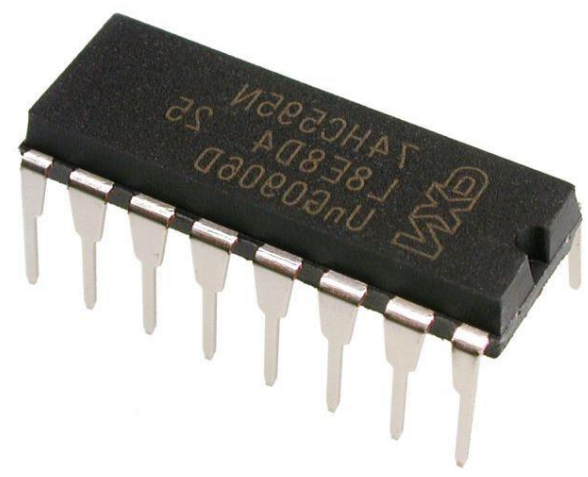

\section{DARLINGTON ULN2803}

Each of the digital i/o pins on the Arduino can only source or sink $40 \mathrm{~mA}$, and pushing the Arduino past these limits may damage it. If you need to get a little more power out of your outputs, try using a Darlington ULN2803. The Darlington chip can source up to $500 \mathrm{~mA}$ of current out each pin, and operates at up to $50 \mathrm{~V}$, so it's beefy enough to drive motors, incandescent bulbs.

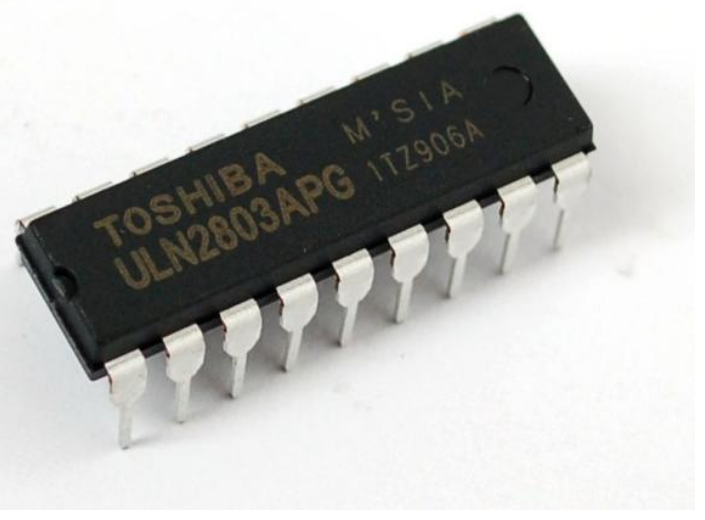

\section{X8 SCROLLING MATRIX LED DISPLAY}

A monochrome (single colour) LED dot matrix display is used for displaying the Characters and Symbols which is interface with a microcontroller.

DC 12 volt to 5 volt converter:

This DC to DC Step-Down Adjustable Power Supply Module is based on the LM2596 switching regulator Input voltage is 4-35V and Output Voltage: 1.5-35V (adjustable). 
Conversion efficiency: Up to $92 \%$ (the higher the voltage, the higher the efficiency) and it is compatible with the following devices Arduino UNO R3, Raspberry Pi, Arduino Mega, Arduino nano, Breadboard power

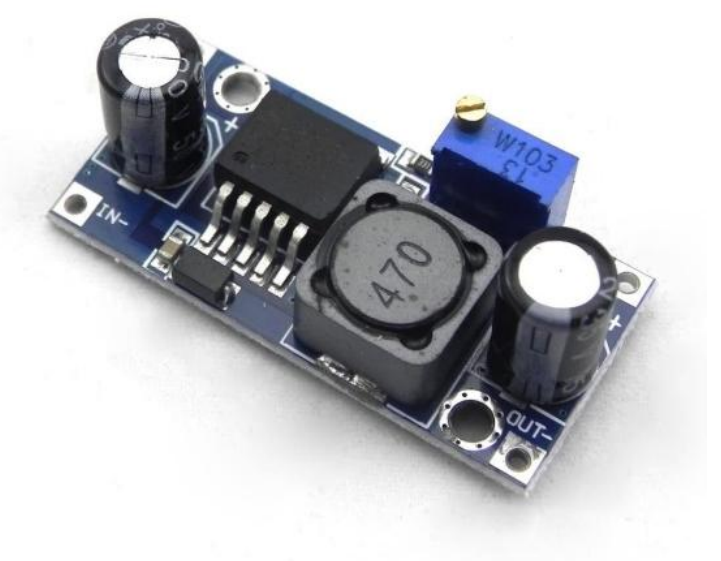

\section{HOW DOES IT WORK?}

Wireless communication technique used in this project is Bluetooth technology. Voice recognition is done in the Android application. User has to install this Android application in his/her smart phone or tablet. Then user has to give voice commands to this android app. Android app then passes these commands to the microcontroller using wireless communication. It means user doesn't have to go near the Electronic notice board to change the scrolling message.Then it passes these commands to the Rolling display which is made up of Matrix LEDs.

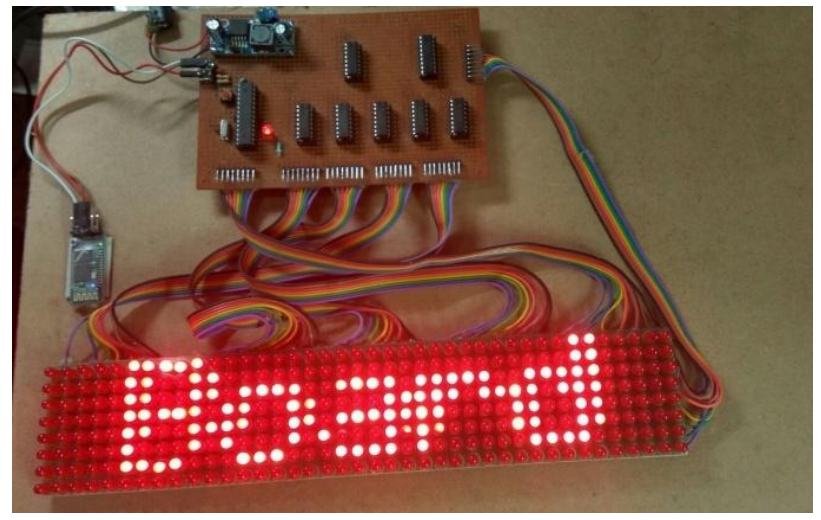

\section{CONCLUSION}

By introducing the concept of wireless technology in the Field of the communication we can make our communication more efficient and faster, with greater efficiency. We can display the messages with less errors and maintenance. This system can be used in college, school, offices, railway station and commercial as well as personal used. The above technical paper explains how we can develop as well as modify voice control Android based wireless notice board. Wireless operations permit services, such as long range communications, that are impossible or impractical to implement with the use of wires. It provides fast transfer of information and are cheaper to install and maintain. It also provides user authentication in order to avoid any misuse of proposed system. With the development of software and hardware capabilities of mobile devices, there is an increased need for device-specific content, what resulted in market changes. Speech recognition technology is of particular interest due to the direct support of communications between human and computers.

The same principle can be applied to control the electrical appliances at a distant location. The project can be further enhance to control different electrical application. 


\section{REFERENCES}

[1] 1Abhishek Gupta, 2Rani Borkar, 3Samita Gawas, 4Sarang Joshi GSM BASED WIRELESS NOTICE BOARD International Journal of Technical Research and Applications e-ISSN: 2320-8163, www.ijtra.com Special Issue 40 (KCCEMSR) (March 2016), PP. 30-33

[2] Neenu Ann George, Prabitha.P, Priyanka.A.K, Ershad.S.B "Raspberry Pi Based Speech Recognition Sensed Smart Notice Board Display", IJSRD International Journal for Scientific Research \& Development| Vol. 3, Issue 12, 2016 | ISSN (online): 2321-0613

[3] Ramchandra K. Gurav, "Wireless Digital Notice Board Using GSM Technology", International Research Journal of Engineering and Technology (IRJET) Volume: 02 Issue: 09 | Dec-2015

[4] Foram Kamdar, "Display Message on Notice Board using GSM" Advance in Electronic and Electric Engineering. ISSN 2231-1297, Volume 3, Number 7 (2013), pp. 827-832

[5] J. S. Lee and Y. C. Huang, "ITRI ZBnode: A ZigBee/IEEE 802.15.4 Plat- form for Wireless Sensor Networks", Proceedings of IEEE International Conference on Systems, Man, and Cybernetics, Taipei, Taiwan, vol. 2, pp. 14621467, October 2006

[6] Safaric, S.; Malaric, K;Zigbee Wireless Standard, IEEE International conference on Multimedia Processing and Communications, March 2006.

[7] Wireless Networking Basics by NETGEAR, Inc. 4500 Great America Parkway Santa Clara, CA 95054 USA. [2] A Message Proliferation System using Short-Range Wireless Devices Department of Information Systems and Media Design,Tokyo Denki University

[8] WIH-Based IEEE 802.11 ECG Monitoring Implementation,Biomedical Engineering Faculty, Sciences and Researches Campus, Azad University, Tehran, Iran [4] PIC Microcontroller and Embedded Systems-By Rolin D. McKinlay, Danny Causey ,Pearson International Edition

[9] Scornavacca, E. Sch. of Inf. Manage., Victoria Univ. Marshall, S. "improving students' learning experience in the classroom through interactive SMS ", Conference Publications on Jan. 2007

[10] Swiatkowski, M. Fac. of Microsyst. Electron. \& Photonics, Wroclaw Univ. of Technol. Wozniak, K. ; Olczyk, L. "Student Notice Board Based on LED Matrix System Controlled over TCP/IP Protocol “, July 2006, Conference Publications.

[11] Li, Xuemei VLSI \& System Laboratory, Beijing University of Technology, China Yuan, Qiuchen ; Wu, Wuchen ; Peng, Xiaohong ; Hou, Ligang , "Implementation of GSM SMS remote control system based on FPGA", Dec. 2010, Conference Publications.

[12] Scornavacca, E. Sch. of Inf. Manage., Victoria Univ. of Wellington, Wellington, New Zealand , "A TwoYear Analysis of Students' Learning Experience Using Interactive SMS in the Classroom", June 2009, Conference Publications

[13] Muhammad Ali Mazidi, Janice G. Mazidi, Rolin D. McKinlay, The 8051 microcontroller and embedded systems using assembly and C, 2nd edition 01-Sep-2007,Pearson Education India.

[14] SMS And MMS Interworking In Mobile Networks Arnaud Henry-Labordère , Artech House mobile communications, 2004 - Technology \& Engineering.

[15] Ayala, Kenneth J. (1996), The 8051 MicrocontrollerArchitecture, Programming and Applications, Delmar Publishers, Inc. India Reprint. [16] GSM telecommunication standards, June 2000 Second edition, European Telecommunications Standards Institute.

[16] Sanjeev Singh, Narendra Singh Pal, Rajat Agarwal, Sharad Yadav, Shubham Bansal, "Android phone speech recognition sensed notice board display", MAT JOURNALS Volume-2, Issue-3

[17] P.S.Sonawane, N.V.Bhamare, S.S.Bothe, S.S.Jadhav, "Rf based wireless notice board",IOSR Journals of computer engineering(IOSR-JCE).

[18] Bhupesh Aneja, Chhavi Srivastav, Kartavya Farashwal, Ajey Aditya, "Wireless Electronic Notice Board Using GSM Technology", ”,International Journal of Advanced Technology in Engineering and Science(IJATES), Volume 4, Issue 3, March 2013.

\section{BIOGRAPHIES}

Rajat Agarwal, student of Electronics and Communication Moradabad Institute of Technology, Moradabad, Uttar Pradesh, and area of interest VLSI Design.

Sanjeev Singh, student of Electronics and Communication Moradabad Institute of Technology, Moradabad, Uttar Pradesh, and area of interest Embeded system and telecommunication.

Sharad Yadav, student of Electronics and Communication Moradabad Institute of Technology, Moradabad, Uttar Pradesh, and area of interest Embedded system and telecommunication.

Shubham Bansal, student of Electronics and Communication Moradabad Institute of Technology, Moradabad, Uttar Pradesh, and area of interest VLSI Design 